\title{
Influence of copper nanoparticle film processing temperature on their structure and electrical properties
}

\author{
N. N. Begletsova ${ }^{1,2}$, A. J. K. Al-Alwani ${ }^{1,3}$, V.S. Atkin ${ }^{1}$, V.P. Sevostyanov ${ }^{2}$, E. G. Glukhovskoy ${ }^{1,2}$ \\ ${ }^{1}$ Saratov State University, Astrakhanskaya, 83, Saratov, 410012, Russia \\ ${ }^{2}$ Scientifc Research Institute of Technology of Organic, Inorganic Chemistry and Biotechnology, \\ Bolshaya Sadovaya, 239, Saratov, 410005, Russia \\ ${ }^{3}$ Babylon University, Babylon, Iraq \\ nadya-beg98@yandex.ru, ammarhamlet2013@yahoo.com,ceba91@list.ru, \\ vsevp@mail.ru, glukhovskoy@gmail.com
}

\begin{abstract}
This work is devoted to the study of the influence of the additional processing at 100,200 and $300{ }^{\circ} \mathrm{C}$ on the morphology, microrelief, elemental composition of the surface and the electrophysical properties of glass/ITO/copper nanoparticle film structures. Studies have shown that with an increase in the processing temperature of the investigating samples reduces the amount of organic matter protecting the copper particles from oxidation. The conductivity of copper nanoparticles increases. The morphology of the surface and the elemental composition of the samples were studied by scanning electron microscopy. The microrelief of the surface and the measurement of the copper nanoparticles current-voltage characteristics were carried out using a scanning probe microscope in atomic force and scanning tunneling microscopy modes.
\end{abstract}

Keywords: Copper nanoparticles, surface morphology and microrelief, elemental composition, current-voltage characteristics.

Received: 27 June 2018

Revised: 21 August 2018

\section{Introduction}

The creation of thin films based on a single component or alternating heterostructures with the desired and controlled properties is one of the more promising directions for the development of modern nanoelectronics. As a component, metallic nanoparticles (NPs), in particular, copper NPs can be used. They are isolated particles with unique physicochemical [1-4] and optical properties $[5,6]$.

Due to their high thermal and electrical conductivity [1,2,7] and mechanical properties [8,9] compared to macro objects copper NPs can be used in solid state and flexible electronics as an alternative to conductive and expensive noble metals such as gold and silver [2,7]. The production of conductive pastes and copper inks for the formation of high conductivity structures in multilayer electronic devices, printed circuit boards, hybrid integrated circuits, photonic crystals are a special areas of application for copper NPs [2,7,10]. They are also used in the composition of lubricants which reduce friction between the contacting surfaces [8]. Recently copper NPs have been actively used as a material for solar cells $[11,12]$. In the near future testing of copper NPs to create metal flexible electrodes of supercapacitors is planned [13].

There are different methods for deposition coating based on copper NPs and their subsequent modification. For example, film deposition in vacuum conditions: chemical vapor deposition (CVD) and physical vapor deposition (PVD) [14, 15], chemical methods of precipitation from solution (Langmuir-Blodgett technology, application in an electric field, etc.) $[16,17]$ are such methods. In this case, the valence state of copper atoms significantly influences the composition, structure and properties of the resulting films. Depending on this, the conditions for their application and the choice of method can be changed. Thus, it was shown in $[14,15]$ that the growth of the monoclinic phase of $\mathrm{CuO}$ begins to predominate over the cubic phase of $\mathrm{Cu}_{2} \mathrm{O}$ with increasing $\mathrm{O} 2$ concentration in the gas mixture of the plasma-chemical reactor. For a liquid medium (phase) the change in the degree of oxidation can be carried out by shifting the $\mathrm{pH}$ of the subphase $[16,17]$. As reported by the authors, the valence of copper ions changed in accordance with a change in the acidity of the aqueous solution, which led to the formation of monolayers and films of different composition and crystal structure.

Copper NPs have the potential of being introduced in electronics. In this area stability and size of particles are important parameters for the practical use them. The stability of copper NPs is important. The stability of the resulting structure or film depends on it. To protect the copper NPs surface from oxidation various stabilizers are used. The use of surfactant leads to a reduction in the aggregation of metal particles. The use of surfactant, along with preventing oxidation of the particle surfaces in air, leads to reduce in their electrical conductivity $[5,18,19]$. 
Thus, there is a dilemma. At the technological stage, the surfactant stabilizer must be removed from the finished products. The modification of the finished films can be different: ultraviolet (UV) treatment, dissolution, chemical transformation, evaporation in an inert atmosphere and temperature annealing in the presence of oxygen. The use of high treatment temperatures (annealing) in the range of $200-300{ }^{\circ} \mathrm{C}$ promotes the decomposition and evaporation of the organic layer followed by fusing the metal particles into a single continuous film. In the literature there is information about the dependence of melting temperature on the dimensions of the metal NPs [20], which is directly related to the ratio of surface and bulk energies. But the relationship "melting temperature-particle size" is non-trivial for the case when the surface of the NPs is surfactant-stabilized. This question is a great practical interest. Although it is still unexplored, any data on the heat treatment and transformation of a complex system of copper NPs in the organic matrix in the literature are represented by single articles.

The aim of this work is to study the influence of the temperature of additional processing at 100,200 and $300{ }^{\circ} \mathrm{C}$ of glass/ITO/copper NPs samples on the morphology, microrelief, elemental composition of the surface and the electrophysical properties of film structures on their basis.

\section{Experimental}

In this work, copper NPs stabilized by surfactant anionic type sodium dodecyl sulfate (SDS) were investigated. The particles were obtained by using the chemical reduction method in micellar solutions of surfactants according to the previously developed procedure [18] at a $\mathrm{pH}$ of 11.0 and the molar ratio of the precursor (copper chloride $\mathrm{CuCl}_{2} \cdot 2 \mathrm{H}_{2} \mathrm{O}$ ) and the reducing agent (hydrazine hydrate $\mathrm{N}_{2} \mathrm{H}_{4} \cdot \mathrm{H}_{2} \mathrm{O}$ ) equal to $1: 150$.

Before depositing the solution of copper NPs on glass substrates with ITO (Indium Tin Oxide) layer in the form of electrodes sample preparation of the suspension obtained by centrifugation on an Elmi CM 70M centrifuge (Latvia) and decanting the solution to remove excess surfactant was carried out. For this purpose in an eppendorf volume of $2.0 \mathrm{ml}, 1.5 \mathrm{ml}$ of a suspension of copper NPs and $0.5 \mathrm{ml}$ of distilled water were placed. Then, for 5 minutes at $5000 \mathrm{rpm}$ the above solution was centrifuged. Then, $1.5 \mathrm{ml}$ of the centrifuged solution was taken out of the eppendorf and the same amount of distilled water was added. Then, for 5 minutes at $5000 \mathrm{rpm}$, the above solution was centrifuged. The $1.5 \mathrm{ml}$ of the centrifuged solution was again removed by decantation from the eppendorf and the same amount of distilled water was added and centrifuged under the foregoing conditions. After $1.5 \mathrm{ml}$ of the solution was removed by decantation. Within 5 minutes the remaining $0.5 \mathrm{ml}$ was again centrifuged. The resulting solution with copper NPs deposited on the bottom of the eppendorf was applied to a glass/ITO substrate with a track using a BIOHIT Proline dispenser. The samples were dried at room temperature for 16 hours.

Investigations of surface morphology and elemental composition of samples with copper NPs on solid substrates were performed on a scanning electron microscope (SEM) of Tescan Mira II LMU (Czech Republic) equipped with an energy-dispersive microanalysis system INCA Energy 350 using a secondary electron detector (SE - second electron) at an accelerating voltage $20-30 \mathrm{kV}$ and pressure in the chamber $5.0-6.0 \cdot 10^{-2} \mathrm{~Pa}$. Surface microrelief and electrophysical properties of samples with copper NPs were studied on the basis of the scanning probe microscope (SPM) NanoEducator II (NT-MDT, Russia) in the modes of atomic force and scanning tunneling microscopy (AFM and STM), respectively. Scanning of samples by the AFM method was carried out in a semi-contact mode at various locations on the surface with a scanning region of $5 \times 5 \mu \mathrm{m}^{2}$. The recording of current-voltage characteristics (CVC) was performed by using STM at various points of the samples surface under study. The amplitude of the alternating voltage was set equal to \pm properties $3 \mathrm{~V}$.

Studies of the film structures for samples with copper NPs were carried out before and after the temperature treatment. For temperature treatment tiles ICA RCT basic (Germany) was used. Its temperature was controlled and kept constant at 100,200 or $300{ }^{\circ} \mathrm{C}$. The accuracy of automatic temperature maintenance was $\pm 5{ }^{\circ} \mathrm{C}$. The sample was annealed at the prescribed temperatures for 10 minutes. After that, it was cooled to room temperature. Then the morphology, surface microrelief, elemental composition and electrophysical properties were studied by SEM, AFM and STM methods.

\section{Results and discussion}

AFM images of the surface of a glass/ITO/copper NPs sample are shown in Fig. 1. In these images, the profile of the surface areas was investigated and the average roughness $(\mathrm{Ra})$ of the sample surface was determined. The choice of research areas was carried out in such a way that they contained objects of fairly uniform height [21]. In AFM images it is difficult to visually identify individual copper NPs. From the analysis of the images in Fig. 1 shows that with an increase in the values of the temperature treatment from room temperature to $300{ }^{\circ} \mathrm{C}$ there is an improvement in the visualization (sharpness of the copper particle faces) of the surface of the film under study. 


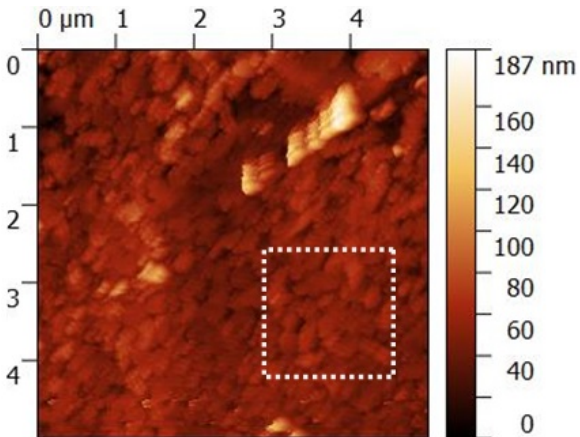

(a)

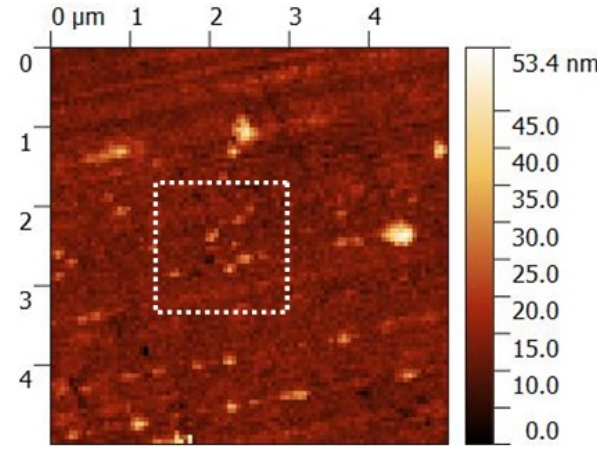

(c)

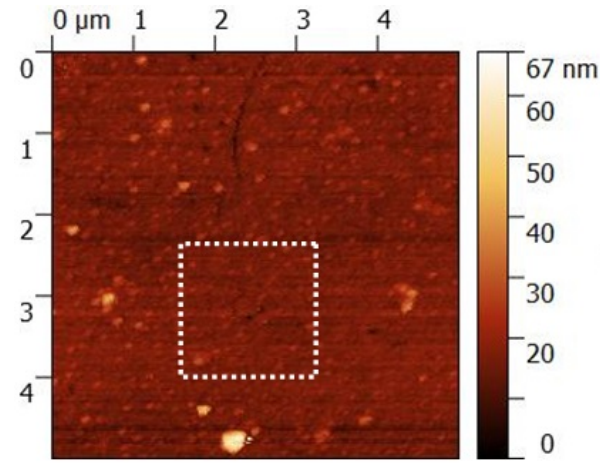

(e)

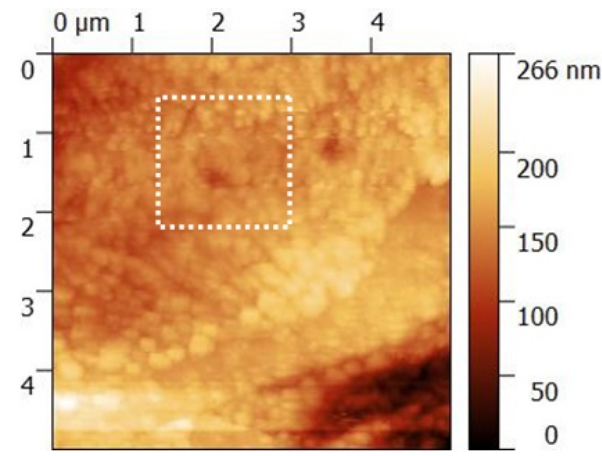

(g)

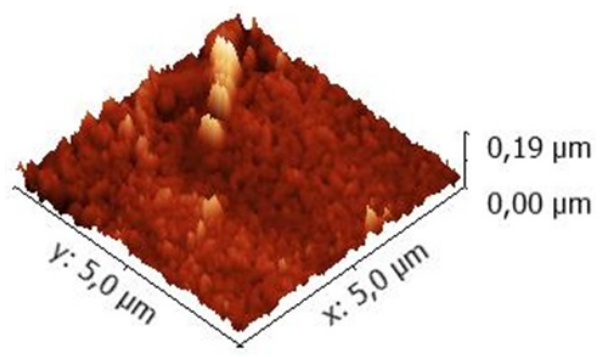

(b)

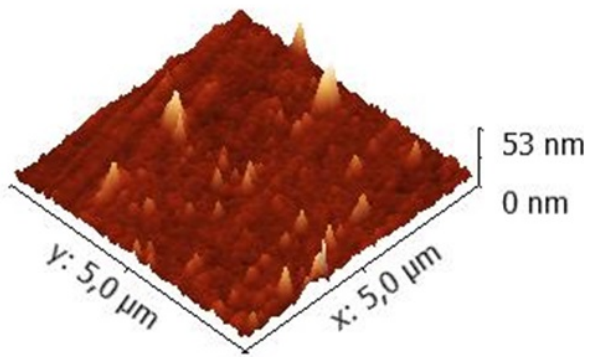

(d)

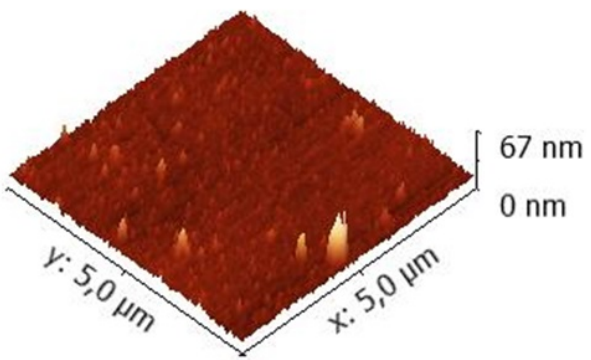

(f)

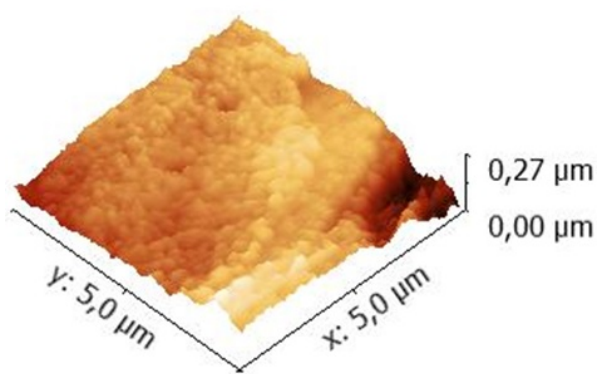

(h)

FIG. 1. 2D (a, c, e, g) and 3D (b, d, f, h) AFM images of the surface of copper NPs on glass/ITO substrates without temperature treatment $(\mathrm{a}, \mathrm{b})$ and after temperature treatment at $100{ }^{\circ} \mathrm{C}(\mathrm{c}, \mathrm{d})$, $200{ }^{\circ} \mathrm{C}(\mathrm{e}, \mathrm{f}), 300{ }^{\circ} \mathrm{C}(\mathrm{g}, \mathrm{h})$. Dashed sections are areas of study of the average surface roughness of the sample 
The reason may be the evaporation of the organic layer of the surfactant SDS, under which there are both separate copper NPs and their aggregates. The obtained images show that the height of the surface microrelief varies in the range from 53 to $270 \mathrm{~nm}$.

The study showed that the average roughness of the sample strongly depends on the temperature (Table 1, Fig. 2,). Increasing the thermal treatment temperature from 23 to $300{ }^{\circ} \mathrm{C}$ leads to a reduction in the average surface roughness from 59 to $30 \mathrm{~nm}$.

TABLE 1. The change in the average roughness of the sample without and after the temperature treatment

\begin{tabular}{|c|c|c|c|c|}
\hline Processing temperature, ${ }^{\circ} \mathbf{C}$ & 23 & 100 & 200 & 300 \\
\hline $\boldsymbol{R}_{\boldsymbol{a}}, \mathbf{n m}$ & 59 & 53 & 30 & 33 \\
\hline
\end{tabular}

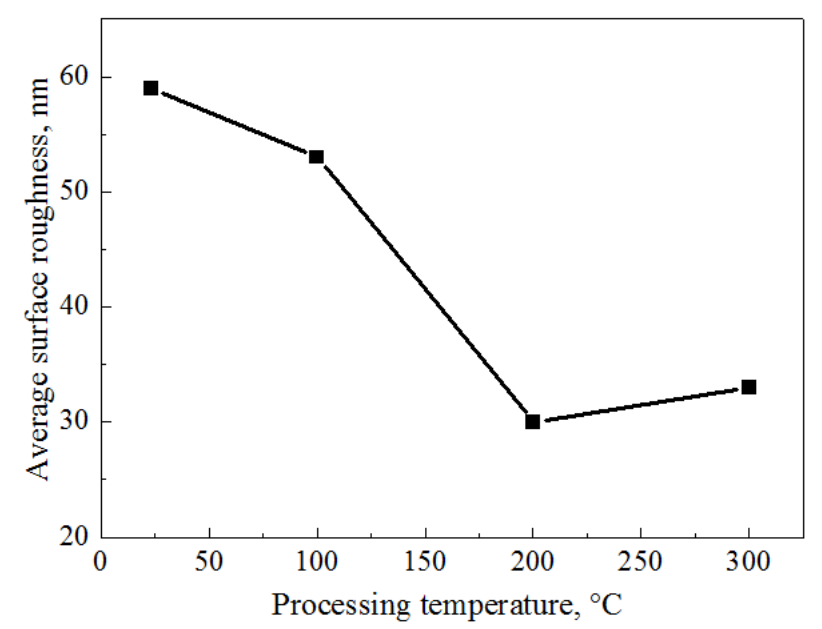

FIG. 2. The dependence of the average roughness of the sample by thermal treatment at 100 , 200 and $300{ }^{\circ} \mathrm{C}$

Such regularities are explained by the following changes in the composition and structure of the resulting film. When the sample is heated to $200-300{ }^{\circ} \mathrm{C}$ with surfactant molecules, a number of changes occur (melting at $206{ }^{\circ} \mathrm{C}$ and decomposition at $216^{\circ} \mathrm{C}$ ). As a result, such transformations lead to a loss of the stabilizing shell of the NPs, their mutual approach to each other and, as a consequence, to reduce in the roughness and an increase in the conductivity of the film, which will be discussed below.

Investigations of surface morphology and element composition of the glass/ITO/copper NPs sample without and after temperature treatment at 100, 200 and $300{ }^{\circ} \mathrm{C}$ showed that irregularly shaped objects with sizes from 1 to $50 \mu \mathrm{m}$ are visible on the surface, as well as objects in the form of rods which are polygons turned by a face to the substrate, a length of 6 to $26 \mu \mathrm{m}$ and a width of 3 to $6 \mu \mathrm{m}$ (Fig. 3(a)). When studying objects in an enlargement scale of $10 \mathrm{kx}$ (Fig. 3(b)), accumulations of predominantly spherical copper particles with dimensions from 30 to $140 \mathrm{~nm}$ in objects with a shape in the form of irregular polygons were detected. The composition was confirmed by energy-dispersive microanalysis. Investigation of the elemental composition of the sample was carried out at various places on its surface (Fig. 4(a)). From the analysis of the obtained data (Table 2) it follows that copper is contained predominantly in polygons of irregular shape (Fig. 4(a), points 1 and 2). On the surface of the glass/ITO substrate (Fig. 4(a), point 3) copper was not detected. The maximum value of the copper content is 3.2 atomic percentages.

By increasing the treatment temperature to $100{ }^{\circ} \mathrm{C}$, a morphology modification of the objects' surfaces occurs in the form of irregular polygons (Fig. 3(c, d)) without any noticeable change in the atomic percentages of chemical elements was observed (Fig. 4(b), Table 3). At a processing temperature of $100{ }^{\circ} \mathrm{C}$ the faces and surfaces of the polygons visually acquired a more pronounced relief. This could be due to the process of alloying copper NPs. At the same time, the images of the polygon faces were visually observed in the obtained SEM images (Fig. 3(c, d)).

After increasing the treatment temperature to $200{ }^{\circ} \mathrm{C}$, an insignificant change was observed in the relief of the surface of the studied objects in the form of polygons (Fig. 3(e, f)). In comparison with the temperature of $100{ }^{\circ} \mathrm{C}$, 


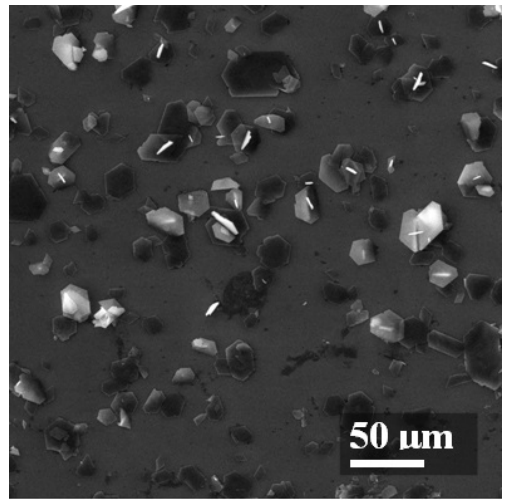

(a)

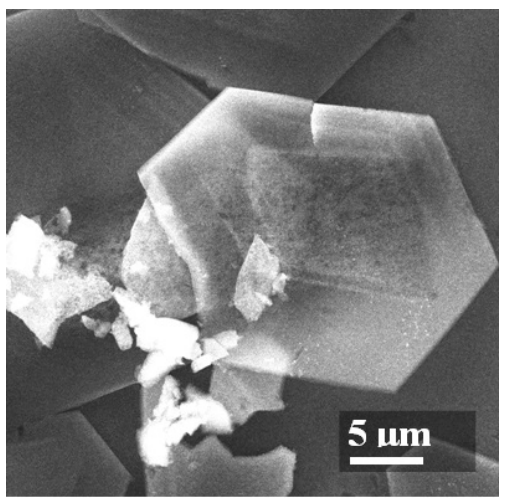

(c)

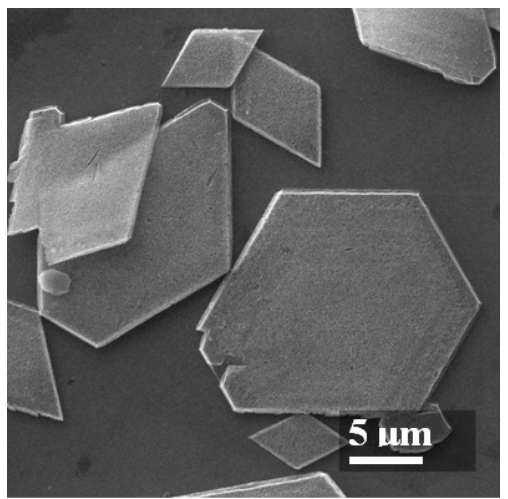

(e)

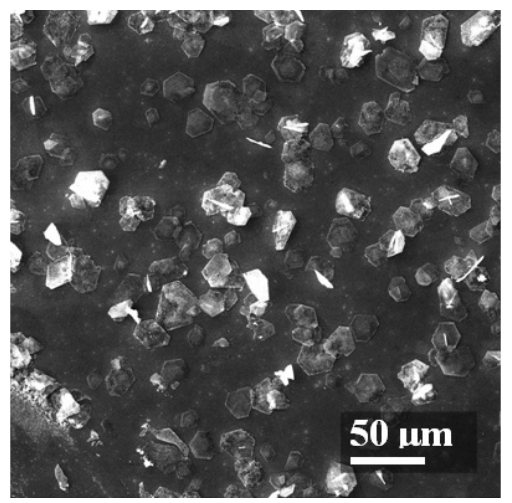

(g)

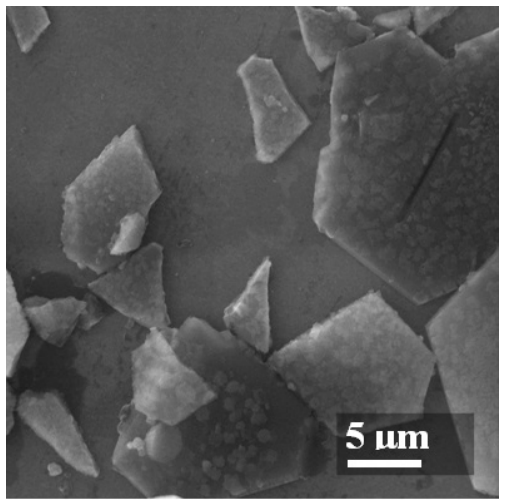

(b)

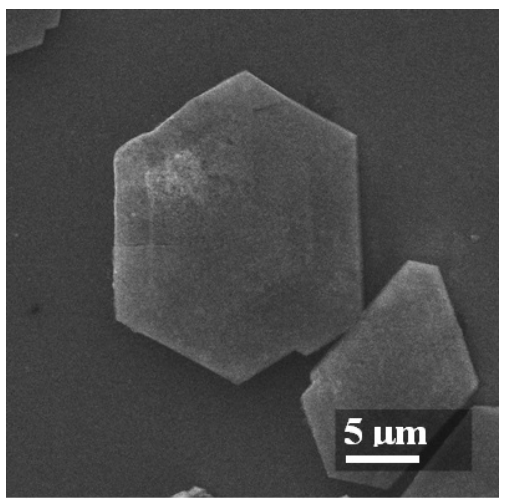

(d)

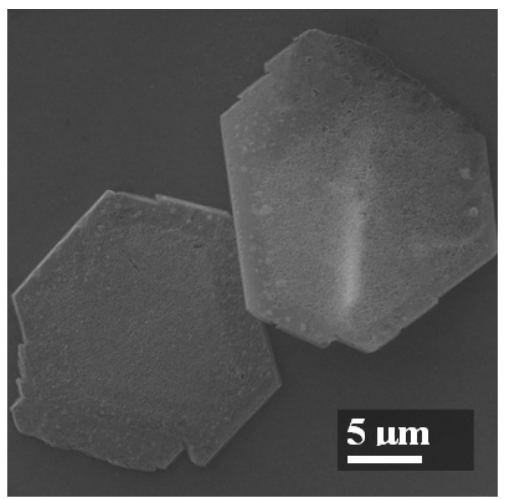

(f)

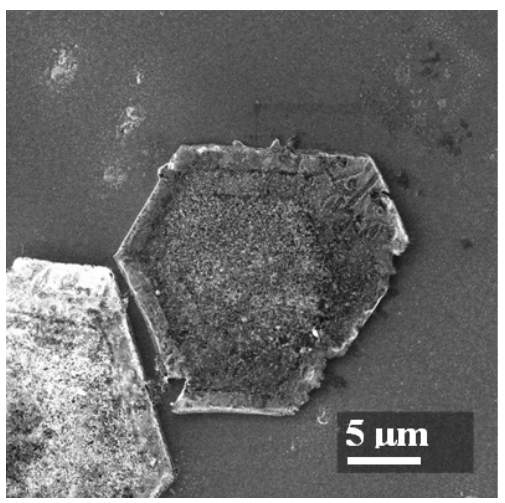

(h)

FIG. 3. SEM images of the surface of copper NPs on glass/ITO substrates without temperature treatment $(\mathrm{a}, \mathrm{b})$ and after temperature treatment at $100{ }^{\circ} \mathrm{C}(\mathrm{c}, \mathrm{d}), 200{ }^{\circ} \mathrm{C}(\mathrm{e}, \mathrm{f}), 300{ }^{\circ} \mathrm{C}(\mathrm{g}, \mathrm{h})$ 


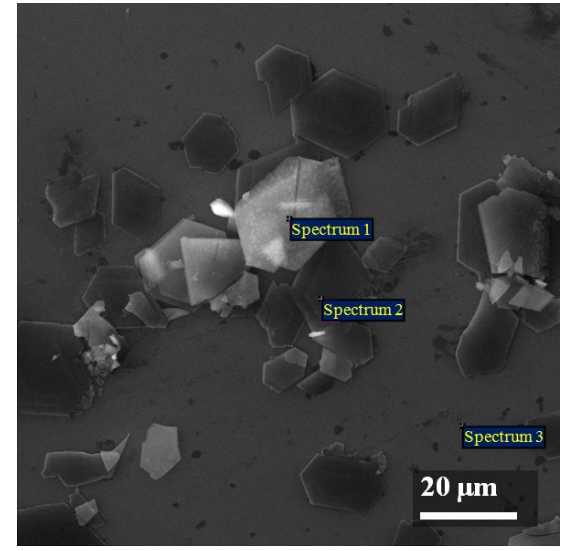

(a)

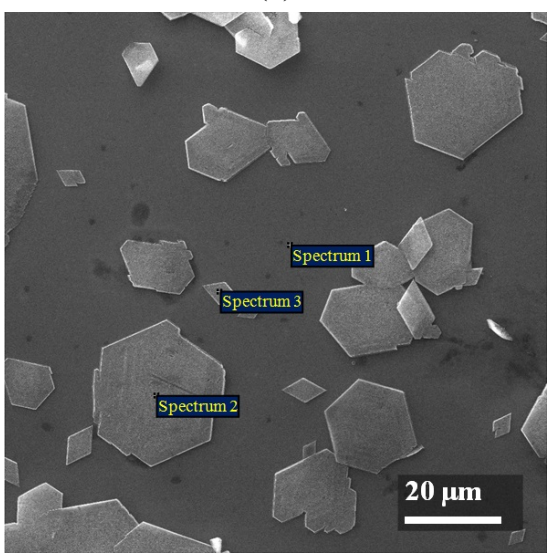

(c)

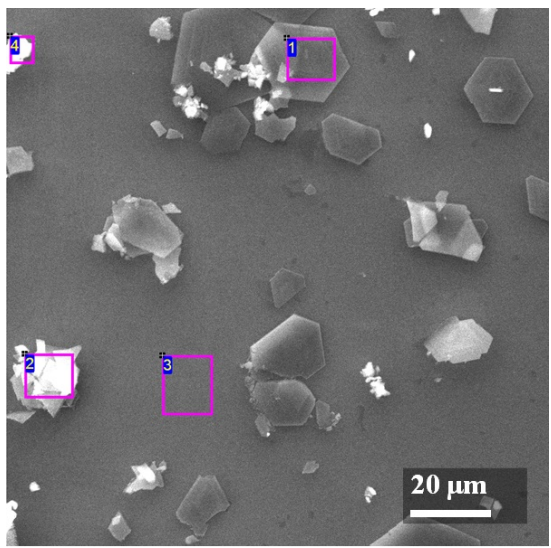

(b)

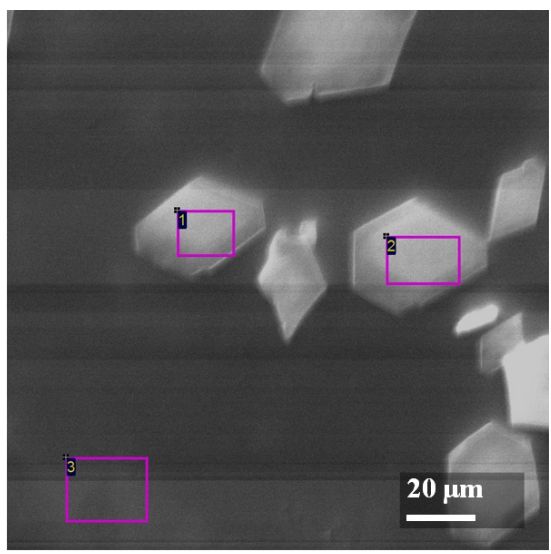

(d)

FIG. 4. SEM images of the surface of copper NPs on glass/ITO substrates without temperature treatment $(\mathrm{a}, \mathrm{b})$ and after temperature treatment at $100{ }^{\circ} \mathrm{C}(\mathrm{c}, \mathrm{d}), 200{ }^{\circ} \mathrm{C}(\mathrm{e}, \mathrm{f}), 300{ }^{\circ} \mathrm{C}(\mathrm{g}, \mathrm{h})$

TABLE 2. The elemental composition of the sample without temperature treatment (all results are given in atomic percentages)

\begin{tabular}{|c|c|c|c|c|c|c|c|c|c|c|c|c|c|c|}
\hline Spectrum & $\mathbf{C}$ & $\mathbf{N}$ & $\mathbf{O}$ & $\mathbf{N a}$ & $\mathbf{M g}$ & $\mathbf{A l}$ & $\mathbf{S i}$ & $\mathbf{C l}$ & $\mathbf{K}$ & $\mathbf{C a}$ & $\mathbf{C u}$ & $\mathbf{I n}$ & Sn & Result \\
\hline $\mathbf{1}$ & 18.8 & 28.1 & 35.0 & - & 0.5 & 0.3 & 10.6 & 0.4 & - & 2.0 & 3.2 & 1.1 & - & 100.0 \\
\hline $\mathbf{2}$ & 23.3 & 28.3 & 30.2 & - & 0.3 & - & 10.8 & 0.2 & 0.2 & 2.9 & 2.6 & 1.2 & - & 100.0 \\
\hline $\mathbf{3}$ & - & 10.4 & 47.0 & 5.3 & 1.5 & 0.8 & 28.2 & - & 0.6 & 4.4 & - & 1.5 & 0.3 & 100.0 \\
\hline
\end{tabular}

TABLE 3. The elemental composition of the sample after the temperature treatment at $100{ }^{\circ} \mathrm{C}$ (all results are given in atomic percentages)

\begin{tabular}{|c|c|c|c|c|c|c|c|c|c|c|c|c|c|c|}
\hline Spectrum & $\mathbf{C}$ & $\mathbf{N}$ & $\mathbf{O}$ & $\mathbf{N a}$ & $\mathbf{M g}$ & $\mathbf{A l}$ & $\mathbf{S i}$ & $\mathbf{C l}$ & $\mathbf{K}$ & $\mathbf{C a}$ & $\mathbf{C u}$ & $\mathbf{I n}$ & Sn & Result \\
\hline $\mathbf{1}$ & 17.1 & 29.9 & 38.3 & - & - & 0.2 & 8.7 & 0.2 & - & 1.9 & 2.6 & 1.1 & - & 100.0 \\
\hline $\mathbf{2}$ & 16.1 & 25.3 & 42.1 & 1.8 & 0.6 & - & 9.5 & 0.3 & 0.1 & 1.3 & 2.2 & 0.7 & - & 100.0 \\
\hline $\mathbf{3}$ & - & - & 51.9 & 5.9 & 1.6 & 0.8 & 31.9 & - & 1.0 & 4.7 & - & 1.7 & 0.5 & 100.0 \\
\hline $\mathbf{4}$ & 14.3 & 23.5 & 39.0 & 3.0 & 0.8 & 0.4 & 13.3 & 0.4 & 0.1 & 1.8 & 2.5 & 0.9 & - & 100.0 \\
\hline
\end{tabular}


TABLE 4. The elemental composition of the sample after the temperature treatment at $200{ }^{\circ} \mathrm{C}$ (all results are given in atomic percentages)

\begin{tabular}{|c|c|c|c|c|c|c|c|c|c|c|c|c|c|}
\hline Spectrum & $\mathbf{C}$ & $\mathbf{O}$ & $\mathbf{N a}$ & $\mathbf{M g}$ & $\mathbf{A l}$ & $\mathbf{S i}$ & $\mathbf{C l}$ & $\mathbf{K}$ & $\mathbf{C a}$ & $\mathbf{C u}$ & $\mathbf{I n}$ & $\mathbf{S n}$ & Result \\
\hline $\mathbf{1}$ & 56.2 & 26.1 & 1.9 & 0.6 & 0.2 & 12.0 & - & 0.3 & 1.9 & - & 0.6 & 0.2 & 100.0 \\
\hline $\mathbf{2}$ & 31.5 & 40.4 & - & 0.6 & 0.4 & 16.6 & 0.3 & 0.3 & 3.6 & 4.6 & 1.7 & - & 100.0 \\
\hline $\mathbf{3}$ & 31.1 & 37.7 & 2.7 & 1.0 & 0.3 & 19.7 & 0.3 & 0.4 & 3.2 & 2.3 & 1.3 & - & 100.0 \\
\hline
\end{tabular}

TABLE 5. The elemental composition of the sample after the temperature treatment at $300{ }^{\circ} \mathrm{C}$ (all results are given in atomic percentages)

\begin{tabular}{|c|c|c|c|c|c|c|c|c|c|c|c|c|c|c|}
\hline Spectrum & $\mathbf{C}$ & $\mathbf{N}$ & $\mathbf{O}$ & $\mathbf{N a}$ & $\mathbf{M g}$ & $\mathbf{A l}$ & $\mathbf{S i}$ & $\mathbf{C l}$ & $\mathbf{K}$ & $\mathbf{C a}$ & $\mathbf{C u}$ & $\mathbf{I n}$ & $\mathbf{S n}$ & Result \\
\hline $\mathbf{1}$ & 8.3 & - & 34.2 & - & 1.1 & 0.7 & 27.2 & 0.4 & 1.1 & 7.2 & 9.6 & 8.6 & 1.6 & 100.0 \\
\hline $\mathbf{2}$ & 6.9 & - & 35.5 & - & 0.8 & 0.7 & 26.1 & 0.5 & 1.2 & 6.8 & 11.0 & 8.7 & 1.8 & 100.0 \\
\hline $\mathbf{3}$ & - & 14.1 & 32.3 & 4.7 & 1.5 & 0.9 & 32.1 & 0.2 & 3.4 & 7.8 & - & - & 3.0 & 100.0 \\
\hline
\end{tabular}

they have acquired an even more pronounced surface relief. This could be due to the evaporation of nitrogen (Fig. 4(c), Table 4).

Increasing the treatment temperature to $300{ }^{\circ} \mathrm{C}$ led to a strong surface modification (Fig. 3(g, h)) and a change in the atomic composition of the chemical elements (Fig. 4(d), Table 5). Perhaps this could be due to the decomposition and evaporation of the organic surfactant layer. As a result, the highest porosity of the face surface of the polygons as compared to other values of the temperature treatment is observed. Therefore, the choice of high temperatures for the annealing of film structures was associated with several processes. At a temperature of $100{ }^{\circ} \mathrm{C}$, the organic layer protecting the copper NPs from oxidation begins to melt without evaporation. At processing temperatures of $200-300{ }^{\circ} \mathrm{C}$, the decomposition and evaporation of short-chain hydrocarbon compounds occurs (Figs. 5 and 6, Tables 6 and 7).

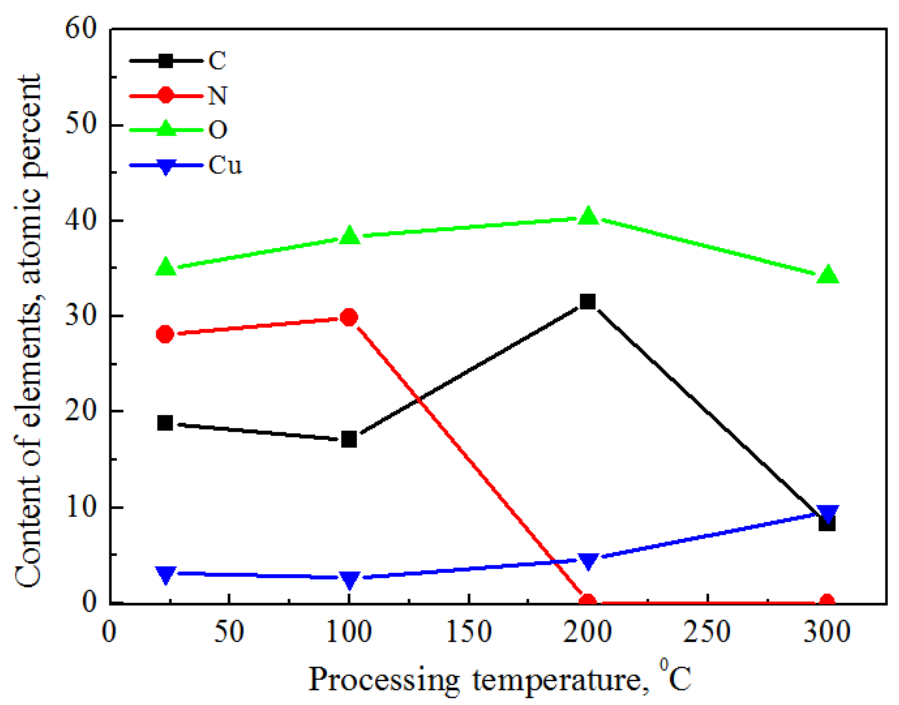

FIG. 5. Dependence of the content of elements $\mathrm{C}, \mathrm{N}, \mathrm{O}, \mathrm{Cu}$ in the composition of the sample after the temperature treatment at 100,200 and $300{ }^{\circ} \mathrm{C}$

From the analysis of the obtained results of the elemental composition of the glass/ITO/copper NPs sample without and after the temperature treatment at 100,200 and $300{ }^{\circ} \mathrm{C}$ a graph of the change in the atomic percentage content of such chemical elements as carbon $(\mathrm{C})$, nitrogen $(\mathrm{N})$, oxygen $\mathrm{O}$ ), copper $(\mathrm{Cu})$ from the values of processing temperatures was constructed (Fig. 5). Elemental composition data are presented in Table 6. 
TABLE 6. The elemental composition of the selected chemical elements in the test sample (all results are given in atomic percentages)

\begin{tabular}{|c|c|c|c|c|}
\hline Processing temperature, ${ }^{\circ} \mathbf{C}$ & $\mathbf{C}$ & $\mathbf{N}$ & $\mathbf{O}$ & $\mathbf{C u}$ \\
\hline $\mathbf{2 3}$ & 18.8 & 28.1 & 35.0 & 3.2 \\
\hline $\mathbf{1 0 0}$ & 17.1 & 29.9 & 38.3 & 2.6 \\
\hline $\mathbf{2 0 0}$ & 31.5 & - & 40.4 & 4.6 \\
\hline $\mathbf{3 0 0}$ & 8.3 & - & 34.2 & 9.6 \\
\hline
\end{tabular}

Figure 6 shows the normalized curve for the selected four elements (Table 6). The normalized elemental composition of $\mathrm{C}, \mathrm{N}, \mathrm{O}, \mathrm{Cu}$ is shown in Table 7.

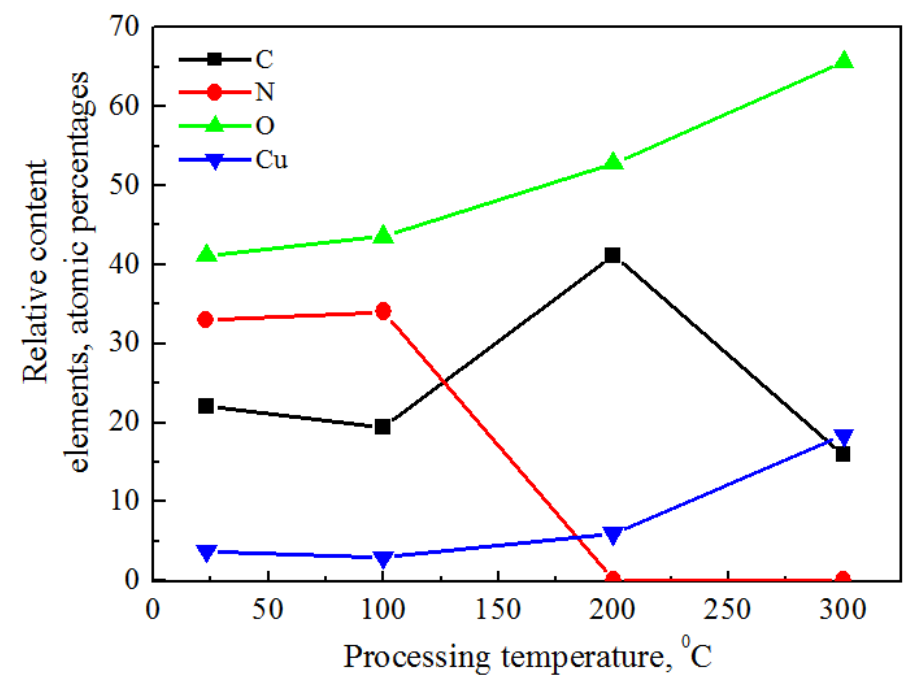

FIG. 6. Dependence of the relative content of elements $\mathrm{C}, \mathrm{N}, \mathrm{O}, \mathrm{Cu}$ in the composition of the sample after the temperature treatment at 100,200 and $300{ }^{\circ} \mathrm{C}$

TABLE 7. The normalized elemental composition of the selected chemical elements in the sample under study (all results are given in atomic percentages)

\begin{tabular}{|c|c|c|c|c|c|}
\hline Processing temperature, ${ }^{\circ} \mathbf{C}$ & $\mathbf{C}$ & $\mathbf{N}$ & $\mathbf{O}$ & $\mathbf{C u}$ & Result \\
\hline $\mathbf{2 3}$ & 22.1 & 33.0 & 41.1 & 3.8 & 100.0 \\
\hline $\mathbf{1 0 0}$ & 19.5 & 34.0 & 43.6 & 3.0 & 100.0 \\
\hline $\mathbf{2 0 0}$ & 41.2 & - & 52.8 & 6.0 & 100.0 \\
\hline $\mathbf{3 0 0}$ & 15.9 & - & 65.6 & 18.4 & 100.0 \\
\hline
\end{tabular}

The results of electrophysical property measurements of a typical glass/ITO/copper NPs sample after the temperature treatment at 100, 200 and $300{ }^{\circ} \mathrm{C}$ in the STM mode is shown in Figs. 7 and 8. As seen from Fig. 7, the CVCs of the tunnel junction are nonlinear and practically symmetrical. When the copper NPs are melted and a continuous metallic layer is formed, the current transfer according to the Ohm's law could be expected. However, the nonlinear dependences of the current on the voltage were experimentally recorded, which may be a consequence of the growth of the oxide film on the surface of the NPs. Copper oxides are semiconductor materials with p-type conductivity, in which the width of the band gap depends on the degree of oxidation. The band gap of copper (I) oxide $\left(\mathrm{Cu}_{2} \mathrm{O}\right)$ is $2.0 \mathrm{eV}$, for copper (II) oxide $(\mathrm{CuO})$ takes values in the range $1.2-1.5 \mathrm{eV}$ [22]. The CVC (Fig. 7) and differential resistances (Fig. 8) show that with increasing values of the temperature processing 
the resistance of the investigated film structure on the basis of the copper NPs reduces. The decomposition and evaporation of the organic layer of a surfactant covering copper particles, which plays the role of an insulator and prevents the tunneling of electrons in the tunnel gap, with increasing the temperature to $200-300{ }^{\circ} \mathrm{C}$, can make the main contribution to the reduction of the resistance of the sample. The results of elemental analysis show that with increasing temperature treatment of the sample the amount of carbon and nitrogen reduced but copper increased (Tables 6 and 7). It is possible that decomposition and evaporation of organic matter contributed to an increase in the conductivity of a film structure based on copper NPs with an increase in temperature processing.

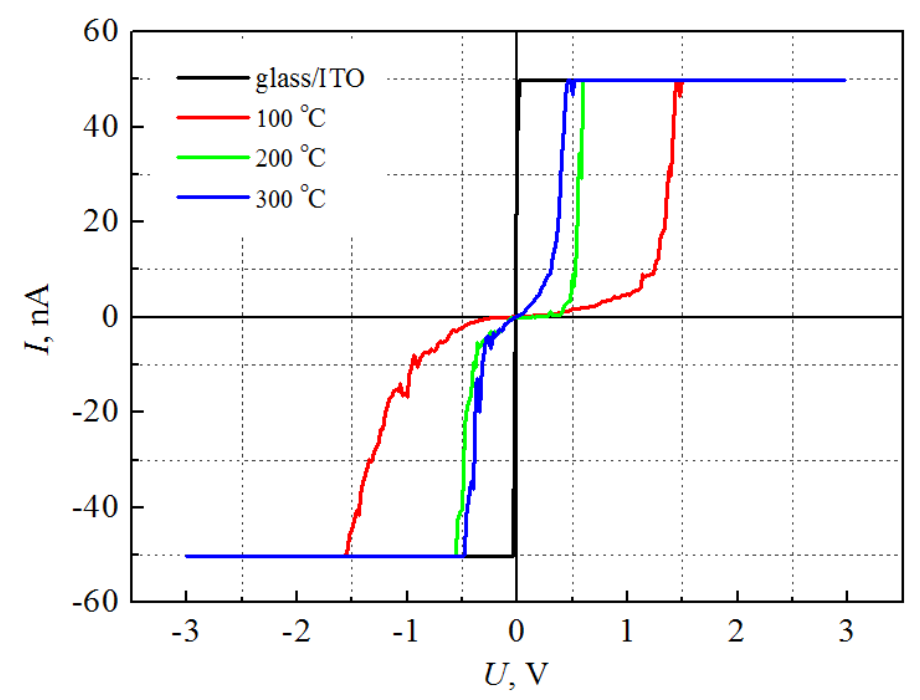

Fig. 7. CVC of sample after heat treatment at 100,200 and $300{ }^{\circ} \mathrm{C}$

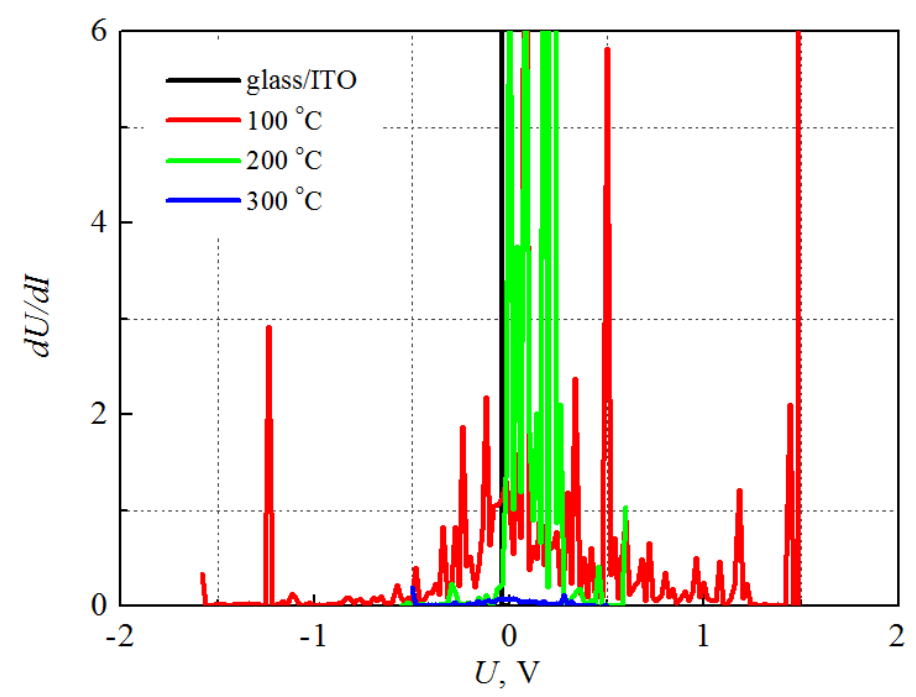

FIG. 8. Differential resistance of the sample after temperature treatment at 100,200 and $300{ }^{\circ} \mathrm{C}$

Figure 9 shows the spectra of the electronic states of the sample after the temperature treatment at 100,200 and $300{ }^{\circ} \mathrm{C}$, reconstructed in the coordinates $f(U)=(d I / d U) /(I / U)$ from the obtained CVC. The data analysis shows that with increasing annealing temperature the band gap reduces from $2.1 \mathrm{eV}$ (for $100{ }^{\circ} \mathrm{C}$ ) to $0.5 \mathrm{eV}$ (for $300{ }^{\circ} \mathrm{C}$ ) (Fig. 9). This can be due to the decomposition and evaporation of the surfactant. This reduces the organic layer of SDS protecting the surface of copper NPs. 


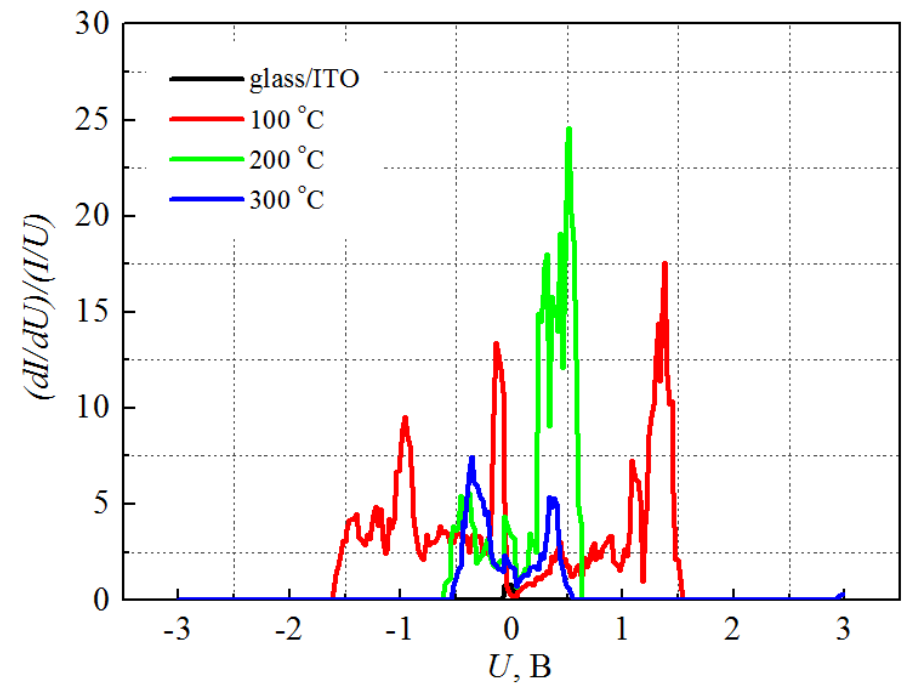

FIG. 9. The spectra of the electronic states of the sample after the temperature treatment at 100 , 200 and $300{ }^{\circ} \mathrm{C}$

\section{Conclusions}

Thus, concerning the influence of additional processing at 100,200 and $300{ }^{\circ} \mathrm{C}$ on the morphology, surface microrelief, element composition and electrophysical properties of glass/ITO/copper NPs samples, SEM, AFM and STM analyses showed that with an increase in the treatment temperature of the substrate there is a modification of the surface and a change in the atomic percentage composition of the chemical elements due to the processes of decomposition and evaporation of the organic layer of the surfactant SDS. In connection with this, the porosity increases for objects having polygon-shaped surface faces. Such polyhedra contain the copper NPs. As a result, with an increase in temperature processing the average surface roughness of the film structure on the basis of copper NPs reduces. In this case, the electrical conductivity of the sample increases. The spectral peaks associated with impurity states inside the forbidden band of the dielectric are reduced.

\section{Acknowledgements}

This work was supported by RFBR grant No. 17-07-00407-a.

\section{References}

[1] Eastman A., Choi S.U.S., Li S., et al. Anomalously increased effective thermal conductivities of ethylene glycolbased nanofluids containing copper nanoparticles. Appl. Phys. Lett., 2001, 78 (6), P. 718-720.

[2] Yabuki A., Arriffin N. Electrical conductivity of copper nanoparticle thin films annealed at low temperature. Thin Solid Films, 2010, 518 (23), P. 7033-7037.

[3] Singh P., Katyal A., Kalra R., et al. Copper nanoparticles in an ionic liquid: an efficient catalyst for the synthesis of bis-(4-hydroxy-2oxothiazolyl)methanes. Tetrahedron Lett., 2008, 49 (4), P. 727-730.

[4] Dragoi B., Ungureanu A., Chirieac A., et al. Structural and catalytic properties of mono- and bimetallic Nickel-Copper nanoparticles derived from $\mathrm{MgNi}(\mathrm{Cu}) \mathrm{Al}-\mathrm{LDHs}$ under reductive conditions. Applied Catalysis A: General, 2015, 504, P. 92-102.

[5] Begletsova N.N., Shinkarenko O.A., Chumakov A.S., et al. Copper nanoparticles obtained by chemical reduction stabilized by micelles of various surfactants. Journal of Physics: Conf. Series, 2017, 917, 092014.

[6] Granata G., Yamaoka T., Pagnanelli F., et al. Study of the synthesis of copper nanoparticles: the role of capping and kinetic towards control of particle size and stability. J. Nanopart. Res., 2016, 18 (133), 12 p.

[7] Li W., Chen M., Wei J., et al. Synthesis and characterization of air-stable $\mathrm{Cu}$ nanoparticles for conductive pattern drawing directly on paper substrates. J. Nanopart. Res., 2013, 15 (1949), 10 p.

[8] Zhou J., Wu Z., Zhang Z., et al. Tribological behavior and lubricating mechanism of Cu nanoparticles in oil. Tribol. Lett., 2000, 8 (4), P. 213-218.

[9] Komeily-Nia Z., Montazer M., Latifi M. Synthesis of nano copper/nylon composite using ascorbic acid and CTAB. Colloids and Surfaces A: Physicochem. Eng. Aspects, 2013, 439, P. 167-175.

[10] Huang K.M., Tsukamoto H., Yong Y., et al. Stabilization of the thermal decomposition process of self-reducible copper ion ink for direct printed conductive patterns. RSC Adv., 2017, 7 (40), P. 25095-25100.

[11] Parveen F., Sannakki B., Mandke M.V., et al. Copper nanoparticles: Synthesis methods and its light harvesting performance. Solar Energy Materials and Solar Cells, 2016, 144, P. 371-382.

[12] Xu B., Zhou J., Ni Z., et al. Synthesis of novel microencapsulated phase change materials with copper and copper oxide for solar energy storage and photo-thermal conversion. Solar Energy Materials and Solar Cells, 2018, 179, P. 87-94. 
[13] Ko Y., Kwon M., Bae W.K., et al. Flexible supercapacitor electrodes based on real metal-like cellulose papers. Nature Communications, 2017, 8 (536), 11 p.

[14] Uschakov A.V., Karpov I.V., Lepeshev A.A., et al. The influence of oxygen concentration on the formation of $\mathrm{CuO}$ and $\mathrm{Cu}_{2} \mathrm{O}$ crystalline phases during the synthesis in the plasma of low pressure arc discharge. Vacuum, 2016, 128, P. $123-127$.

[15] Fedorov L.Yu., Karpov I.V., Ushakov A.V., et al. Study of Phase Composition of CuO/Cu2O Nanoparticles Produced in the Plasma of a Low-Pressure Arc Discharge. Inorganic Materials: Applied Research, 2018, 9 (2), P. 323-328.

[16] Lin B., Bohanon T.M., Shih M.C., et al. X-ray Diffraction Studies of the Effects of $\mathrm{Ca}^{2+}$ and $\mathrm{Cu}^{2+}$ on Langmuir Monolayers of Heneicosanoic Acid. Langmuir, 1990, 6, P. 1665-1667.

[17] Khomutov G.B., Yakovenko S.A., Yurova T.V., et al. Effect of compression of a stearic acid monolayer on interfacial binding of copper ions and cluster formation. Supramolecular Science, 1997, 4, P. 349-355.

[18] Begletsova N., Selifonova E., Chumakov A., et al. Chemical synthesis of copper nanoparticles in aqueous solutions in the presence of anionic surfactant sodium dodecyl sulfate. Colloids and Surfaces A: Physicochemical and Engineering Aspects, 2018, 552, P. 75-80.

[19] Kosolapova K., Al-Alwani A., Gorbachev I., et al. Purification non-aqueous solution of quantum dots CdSe- CdS-ZnS from excess organic substance-stabilizer by use PE-HD membrane. Journal of Physics: Conference Series, 2015, 643, 012084.

[20] Kumar R., Kumar M. Effect of size on cohesive energy, melting temperature and Debye temperature of nanomaterials. Indian J. Pure Appl. Phys., 2012, 50, P. 329-334.

[21] Mikhailov A.I., Kabanov V.F., Gorbachev I.A., et al. Study of the properties of II-VI and III-V semiconductor quantum dots. Semiconductors, 2018, 52 (6), P. 750-754.

[22] Srivastava S., Kumar M., Agrawal A., et al. Synthesis and characterisation of copper oxide nanoparticles. Journal of Applied Physics, 2013,5 (4), P. 61-65. 